University of Nebraska - Lincoln

DigitalCommons@University of Nebraska - Lincoln

January 1995

\title{
Electronic structures and Curie temperatures of iron-based rare- earth permanent-magnet compounds
}

\author{
J.P. Woods \\ University of Nebraska - Lincoln \\ B.M. Patterson \\ University of Nebraska - Lincoln \\ A.S. Fernando \\ University of Nebraska - Lincoln \\ Sitaram Jaswal \\ University of Nebraska, sjaswal1@unl.edu \\ D. Welipitiya \\ University of Nebraska - Lincoln \\ See next page for additional authors
}

Follow this and additional works at: https://digitalcommons.unl.edu/physicssellmyer

Part of the Physics Commons

Woods , J.P.; Patterson, B.M.; Fernando, A.S.; Jaswal, Sitaram; Welipitiya, D.; and Sellmyer, David J., "Electronic structures and Curie temperatures of iron-based rare-earth permanent-magnet compounds " (1995). David Sellmyer Publications. 99.

https://digitalcommons.unl.edu/physicssellmyer/99

This Article is brought to you for free and open access by the Research Papers in Physics and Astronomy at DigitalCommons@University of Nebraska - Lincoln. It has been accepted for inclusion in David Sellmyer Publications by an authorized administrator of DigitalCommons@University of Nebraska - Lincoln. 


\section{Authors}

J.P. Woods , B.M. Patterson, A.S. Fernando, Sitaram Jaswal, D. Welipitiya, and David J. Sellmyer 


\title{
Electronic structures and Curie temperatures of iron-based rare-earth permanent-magnet compounds
}

\author{
J. P. Woods, B. M. Patterson, A. S. Fernando, S. S. Jaswal, D. Welipitiya, and \\ D. J. Sellmyer \\ Behlen Laboratory of Physics and Center for Materials Research and Analysis, University of Nebraska, \\ Lincoln, Nebraska 68588
}

(Received 25 May 1994; revised manuscript received 24 August 1994)

\begin{abstract}
The modification of the electronic structures of $\mathrm{Sm}_{2} \mathrm{Fe}_{17-x} \mathrm{Al}_{x} \mathrm{~N}_{y}, \mathrm{NdFe}_{11} \mathrm{TiN}_{y}$, and $\mathrm{YFe}_{12-x} \mathrm{Mo}_{x}$ upon alloying and nitriding are examined with self-consistent spin-polarized calculations and soft$\mathrm{x}$-ray photoemission measurements between 18 and $135 \mathrm{eV}$. The changes in the Curie temperature $T_{c}$ with substitutional modifications and nitrogen addition are modeled with self-consistent spinpolarized electronic structure calculations and the spin-fluctuation theory of Mohn and Wohlfarth which relates the electronic structure to $T_{c}$. The calculations show that the spin-summed density of states at the Fermi energy is related to $T_{c}$. The photoemission spectra are dominated by the Fe $3 d$ electrons within $3 \mathrm{eV}$ of the Fermi energy in agreement with calculations. Changes in the density of states at the Fermi energy for interstitial and substitutional modification compare well with calculations. Using photoemission results with experimental magnetic moments for the substitutional modification of the compound $\mathrm{Sm}_{2} \mathrm{Fe}_{17-x} \mathrm{Al}_{x}$, the spin-fluctuation theory predicts a change in $T_{c}$ in agreement with the measured change in $T_{c}$. Spin-resolved photoemission spectra for $c$-axis oriented $\mathrm{Sm}_{2} \mathrm{Fe}_{17} \mathrm{~N}_{2.6}$ with magnetization perpendicular to the surface are presented and compared to theoretical calculations.
\end{abstract}

\section{INTRODUCTION}

The magnetic properties required for permanent magnetic materials are large magnetization, large uniaxial magnetic anisotropy, and high Curie temperature $\left(T_{c}\right)$. Since the $1960 \mathrm{~s}$, superior magnetic materials are compounds of light rare-earth elements $(R)$ and ferromagnetic transition metals $(T) .{ }^{1}$ The $R-T$ compounds have a large magnetization due to the high $T$ concentration and a large magnetic anisotropy due to the anisotropic crystal structure and large single-ion anisotropy associated with non $s$-state rare earths. The first $R-T$ compound to attain widespread application was $\mathrm{SmCo}_{5}$ having the $\mathrm{CaCu}_{5}$ structure. $^{2}$ The SmCo-based materials are inherently expensive due to the high cost of the raw materials. Iron-based $R-T$ compounds are less expensive but compounds with larger Fe concentrations have lower $T_{c}$ values. ${ }^{3}$ Initial work with rapidly quenched $R-T$ systems alloyed with boron led to the discovery of the ternary crystalline compound $\mathrm{Nd}_{2} \mathrm{Fe}_{14} \mathrm{~B}$ (Ref. 1) with $T_{c}=585$ K.

Coey and co-workers ${ }^{4}$ recently have shown that interstitial nitrogen addition to the $R_{2} \mathrm{Fe}_{17}$ compounds increases $T_{c}$ by as much as $400 \mathrm{~K}$. The $R_{2} \mathrm{Fe}_{17}$ compounds have planar magnetic anisotropy for all the rare earths, and only the compound $\mathrm{Sm}_{2} \mathrm{Fe}_{17} \mathrm{~N}_{2.6}$ has a uniaxial magnetic anisotropy. The nitrided iron-rich compound $\mathrm{Sm}_{2} \mathrm{Fe}_{17} \mathrm{~N}_{2.6}$ with $T_{c}=743 \mathrm{~K}$ is being developed for permanent-magnet applications.

The $R$-Fe compounds can also be modified with partial substitution, for example, $\mathrm{Al}$ for $\mathrm{Fe}$ in the compound $\mathrm{Sm}_{2} \mathrm{Fe}_{17-x} \mathrm{Al}_{x} \cdot{ }^{5-8}$ Interestingly, $T_{c}$ increases for Al substitution for values of $x$ up to 3 while the magnetiza- tion per formula unit decreases. Partial substitution of a non-magnetic transition metal $(M)$ for Fe stabilizes the $R \mathrm{Fe}_{12-x} M_{x}$ structure in the $\mathrm{ThMn}_{12}$ structure. The 2:17 and 1:12 structures are closely related as slight modifications of the $\mathrm{CaCu}_{5}$ hexagonal structure. ${ }^{9}$ In considering possible compounds for examining changes in $T_{c}$ with $M$ substitution, the compound $\mathrm{YFe}_{12-x} \mathrm{Mo}_{x}$ was selected because it exhibits a large decrease in $T_{c}$ with increased Mo concentration ${ }^{10}$ and the substitution site has been determined by x-ray diffraction. ${ }^{11}$

In this work we have investigated the mechanism for the drastic change in the $T_{c}$ in the $R$-Fe compounds with interstitial addition and substitutional modification. Previous workers ${ }^{6,10,12-14}$ have advocated a mean-field model to explain qualitatively the increase in $T_{c}$ with lattice expansion. In Sec. II the inadequacies of this model are discussed and the spin-fluctuation theory of Mohn and Wohlfarth ${ }^{15}$ is summarized. In Sec. III selfconsistent, spin-polarized calculations of the electronic structure for several Fe-based compounds and quantitative comparisons of the theoretical and experimental change in $T_{c}$ with modifications are presented. In Sec. IV the details of sample preparation, characterization, and photoemission results are presented and discussed. The conclusions of this work are summarized in Sec. V.

\section{CURIE TEMPERATURE MODELS}

Previous investigators have explained the variation of $T_{c}$ with interstitial addition ${ }^{12-14}$ and substitutional modification ${ }^{6,10}$ with a mean-field model and an interatomic-distance-dependent exchange energy as represented by the Bethe-Slater curve. As noted by Herbst, ${ }^{1}$ 
"attributing $T_{c}$ behavior solely to distance dependent exchange is undoubtedly an oversimplification." Although the distance-dependent exchange model does correlate the results of $T_{c}$ variation in a wide variety of the compounds, there are several weaknesses of the model that must be considered: (1) Goodenough ${ }^{16}$ has discussed the Bethe-Slater curve and concludes that the curve is incorrect. (2) $T_{c}$ depends on the local coordination of the magnetic ions. In simple systems with one site, the theory is straightforward but with three or more Fe sites in the $R$-Fe compounds the effect of local coordination is complicated, especially with substitutional modifications. (3) As noted by Chen, ${ }^{17}$ the mean-field model is an ionic model which applies well to the rare-earth elements but Fe-based compounds are clearly itinerant magnetic systems. (4) There are several compounds that do not exhibit an increase in $T_{c}$ as the lattice constant increases. For the compound $\mathrm{YFe}_{12-x} \mathrm{Mo}_{x},{ }^{10}$ as $x$ increases the lattice constant increases but $T_{c}$ decreases. As a second example, a recent publication ${ }^{18}$ has shown that for the Sisubstituted compound $\mathrm{Ce}_{2} \mathrm{Fe}_{17-x} \mathrm{Si}_{x}$, as $x$ increases, the volume decreases, and the magnetic moment per formula unit decreases, but $T_{c}$ increases. The mean-field model does have its merits, but the underlying mechanism that alters $T_{c}$ in the modified $R$-Fe compounds is not readily apparent in the mean-field model of magnetism.

Mohn and Wohlfarth ${ }^{15}$ have developed a model that considers the spin fluctuations of local atomic moments in determining $T_{c}$ in itinerant electron systems. $T_{c}$ is determined by the solution to the algebraic equation

$$
\frac{T_{c}^{2}}{T_{S}^{2}}+\frac{T_{c}}{T_{\mathrm{SF}}}-1=0,
$$

where $T_{S}$ is the calculated Curie temperature based on the Stoner model of itinerant electron magnetism and $T_{\mathrm{SF}}$ is a characteristic temperature describing the influence of spin fluctuations. The calculated value of $T_{S}$ is much larger than the measured $T_{c},{ }^{15}$ and $T_{c}$ is determined primarily by the second term in Eq. (1). The spin-fluctuation temperature is given by ${ }^{15}$

$$
T_{\mathrm{SF}}=\frac{M_{0}^{2}}{10 k_{B} \chi_{0}},
$$

where $M_{0}$ is the zero-temperature magnetic moment and $k_{B}$ is Boltzmann's constant. $\chi_{0}$ is the exchange-enhanced susceptibility and is given by ${ }^{19}$

$$
\frac{1}{\chi_{0}}=\frac{1}{4 \mu_{B}^{2}}\left(\frac{1}{N_{\uparrow}\left(E_{F}\right)}+\frac{1}{N_{\downarrow}\left(E_{F}\right)}-2 I\right),
$$

where $N_{\uparrow}\left(E_{F}\right)$ and $N_{\downarrow}\left(E_{F}\right)$ are the up- and down-spin density of states at the Fermi energy and $I$ is the Stoner parameter. The quantities $M_{0}, \chi_{0}, N_{\uparrow}\left(E_{F}\right), N_{\downarrow}\left(E_{F}\right)$, and $I$ are directly determined by theoretical electronic structures.

\section{THEORETICAL ELECTRONIC STRUCTURE}

The electronic structures of $\mathrm{Y}_{2} \mathrm{Fe}_{17}$ and $\mathrm{NdFe}_{11} \mathrm{Ti}$ parent compounds and their nitrides $\mathrm{Y}_{2} \mathrm{Fe}_{17} \mathrm{~N}_{3}$ and $\mathrm{NdFe}_{11} \mathrm{TiN}$ have been reported by Jaswal et $a .^{20,21}$
The electronic structure is calculated using self-consistent spin-polarized calculations based on the linear-muffintin-orbital method in the scalar relativistic approximation. ${ }^{22}$ The theoretical ratio $T_{c}\left(\mathrm{Y}_{2} \mathrm{Fe}_{17} \mathrm{~N}_{3}\right) / T_{c}\left(\mathrm{Y}_{2} \mathrm{Fe}_{17}\right)$ based on the spin-fluctuating theory and the calculated density of states (DOS) is compared to the measured $T_{c}$ ratio. The theoretical $T_{c}$ ratio for the parent compound $\mathrm{Y}_{2} \mathrm{Fe}_{17}(2.34)$ is within $9 \%$ of the experimental value (2.14). ${ }^{20}$ For the $\mathrm{NdFe}_{11} \mathrm{Ti}$ compound, the calculated Curie temperature ratio (1.32) is within $1 \%$ of the experimental value (1.31). ${ }^{21}$ The absolute $T_{c}$ 's are not correctly predicted by the spin-fluctuation theory using the calculated electronic structures, but the calculated relative changes in $T_{c}$ 's are in very good agreement with experiment.

The calculated DOS for $\mathrm{Y}_{2} \mathrm{Fe}_{17} \mathrm{~N}_{3}$ has been employed by Katter et al. ${ }^{23}$ to determine the $T_{c}$ of $\mathrm{Sm}_{2} \mathrm{Co}_{17} \mathrm{~N}_{3}$. The Fermi level is shifted to account for the additional $3 d$ electron on Co and very little change in the spin-resolved DOS at the Fermi energy is interpolated from the calculated electronic structure. ${ }^{20}$ In this case, $T_{c}$ is independent of $\chi_{0}$, and a reduced $T_{c}$ in $\mathrm{Sm}_{2} \mathrm{Co}_{17} \mathrm{~N}_{3}$ is due to a reduction in the magnetic moment. The predicted $T_{c}$ $(834 \mathrm{~K})$ is in very good agreement with the experimental value $(811 \mathrm{~K})$.

The spin-fluctuation theory is applied here to the substitutional modification of $T_{c}$ in $R$-Fe compounds. The self-consistent spin-polarized electronic structure for $\mathrm{Y}_{2} \mathrm{Fe}_{12-x} \mathrm{Mo}_{x}$ for $x=1$ and 2 is shown in Fig. 1. The electronic structure is dominated by the Fe $3 d$ bands near the Fermi energy, and an exchange splitting of about 2.0 $\mathrm{eV}$ is evident. There are only slight changes in the electronic structure as the Mo concentration increases from $x=1$ to $x=2$; the DOS at $E_{F}$ changes less than $3 \%$ in both the up- and down-spin DOS. The calculated and measured zero-temperature magnetic moments are not in good agreement. The magnetic moment per formula unit (f.u.) from the calculations are $22.0 \mu_{B} /$ f.u. and

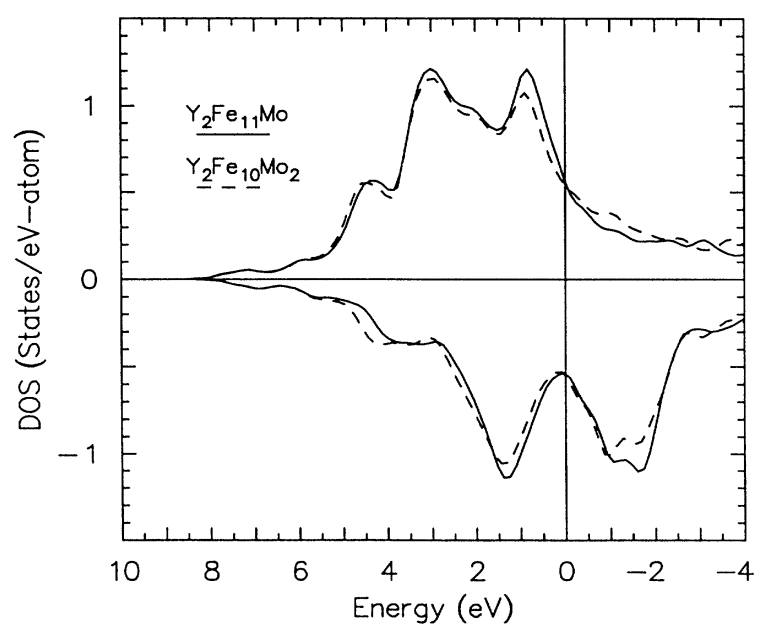

FIG. 1. Spin-polarized density of states for $\mathrm{YFe}_{11} \mathrm{Mo}$ (solid line) and $\mathrm{YFe}_{10} \mathrm{Mo}_{2}$ dashed line. The zero of energy represents the Fermi energy. 
$19.2 \mu_{B} /$ f.u. for $x=1$ and 2 , respectively. Experimentally the moments have been measured ${ }^{10}$ as $23.7 \mu_{B}$ /f.u. and $14.1 \mu_{B} /$ f.u. for $x=1$ and 2 , respectively. The disagreement in the magnetic moment may be due to a noncollinear alignment of the moments in the compound and/or the inadequacies of representing the disorder of the Mo sites in the theoretical model. ${ }^{21}$ Applying the spin-fluctuation model with the calculated $M_{0}$ and $\chi_{0}$ values, the $T_{c}$ ratio is 0.74 which is within $9 \%$ of the measured $T_{c}$. This good agreement between theoretical and experimental $T_{c}$ ratios indicates that the spin-fluctuation theory is a reasonable model for the substitutional modification of these compounds. In our earlier work ${ }^{24}$ using the experimental values of $M_{0}$ and photoemission results, we concluded that the spin-fluctuation theory did not predict the change in $T_{c}$ correctly. The discrepancy is due to the difference in the experimental and theoretical values of $M_{0}$.

In the next section, the electronic structures of several compounds are measured with photoemission spectroscopy. The relevance of the total (spin-integrated) DOS to the spin-fluctuation model is revealed in Fig. 2 by plotting the exchange-enhanced susceptibility $\chi_{0}$ as a function of the total DOS at the Fermi energy $N_{\text {tot }}\left(E_{F}\right)$ obtained from calculations. The data are taken from previous results on the 2:17 (Ref. 20) and 1:12 (Ref. 21) compounds and the present results on the Mo-substituted compounds. A linear dependence is observed for the Febased compounds considered, and the functional form is included on the plot. The parent and nitrided 2:17 compounds are at the limits of the range of values considered, and the change in $T_{c}$ in the interstitial modification is

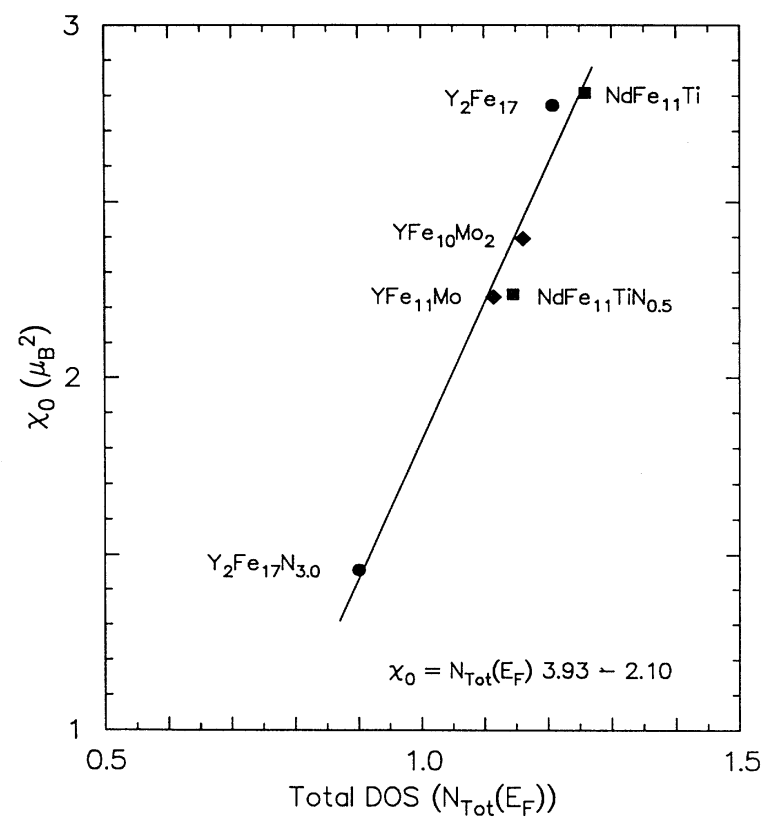

FIG. 2. Calculated results of the enhanced susceptibility $\chi_{0}$ as a function of the total (spin-summed) density of states at the Fermi energy $N_{\text {tot }}\left(E_{F}\right)$. The functional form of the linear fit is indicated on the plot. dominated by the change in the value of $\chi_{0}$. In contrast, the substitutional modification of $\mathrm{Y}_{2} \mathrm{Fe}_{12-x} \mathrm{Mo}_{x}$ shows a relatively small change in $\chi_{0}$, emphasizing the relative importance of the change in $M_{0}$ in the alteration of $T_{c}$. As noted previously, $T_{c}$ increases for Al-substitution in $\mathrm{Sm}_{2} \mathrm{Fe}_{17-x} \mathrm{Al}_{x}$ while the magnetization decreases. Using the experimental values of the magnetic moment and $T_{c}$ for the Al-substituted compounds, the spin-fluctuation theory predicts a dramatic decrease in $\chi_{0}$ which can be used with Fig. 2 to anticipate a similar decrease in the experimentally measured $N_{\text {tot }}\left(E_{F}\right)$. The electronic structure of the Al-substituted compounds have not been calculated because the site of $\mathrm{Al}$ substitution has not been uniquely identified. ${ }^{5,8}$

\section{EXPERIMENTAL RESULTS AND DISCUSSION}

\section{A. Sample preparation}

Bulk samples of $\mathrm{Sm}_{2} \mathrm{Fe}_{17-x} \mathrm{Al}_{x} \mathrm{~N}_{y}, \mathrm{NdFe}_{11} \mathrm{TiN}_{y}$, and $\mathrm{Y}_{2} \mathrm{Fe}_{12-x} \mathrm{Mo}_{x}$ were prepared by arc melting at least $\mathbf{9 9 . 9 \%}$ pure powders in flowing argon on a water-cooled copper boat. The samples were melted several times to ensure homogeneity. The samples were wrapped separately in Ta foil and annealed in a vacuum of $10^{-6}$ Torr for several days at temperatures ranging from 850 $\mathrm{C}$ to $1100^{\circ} \mathrm{C}$; the details of the vacuum annealing can be found in the literature $\left[\mathrm{Sm}_{2} \mathrm{Fe}_{17}\right.$ (Ref. 12), $\mathrm{NdFe}_{11} \mathrm{Ti}$ (Ref. 25), $\mathrm{Y}_{2} \mathrm{Fe}_{12-x} \mathrm{Mo}_{x}$ (Ref. 10)]. A second $\mathrm{Sm}_{2} \mathrm{Fe}_{17}$ sample was prepared by arc melting $\mathrm{Sm}, \mathrm{Fe}$, and $4 \%$ $\mathrm{Nb}$ (by weight) and the vacuum annealing stage was not required to obtain single-phase samples. ${ }^{26} \mathrm{~A}$ portion of each button was pulverized and powder x-ray diffraction verified the proper structure for each compound (rhombehedral $\mathrm{Th}_{2} \mathrm{Zn}_{17}$ for the 2:17 compounds and tetragonal $\mathrm{ThMn}_{12}$ for the 1:12 compounds). A disk approximately $1.5 \mathrm{~cm}$ in diameter and $1 \mathrm{~mm}$ thick was spark cut from each button and the surfaces were polished to an optically smooth finish. Scanning electron micrographs of the $\mathrm{Sm}_{2} \mathrm{Fe}_{17}+\mathrm{Nb}$ sample showed a polycrystalline structure with grain diameters less than $1 \mu \mathrm{m}$. X-ray diffraction of the $\mathrm{Sm}_{2} \mathrm{Fe}_{17}+\mathrm{Nb}$ bulk sample showed that it was textured with the $c$ axis perpendicular to the surface. The texturing may be due to the cooling pattern of the button on the cooled copper boat. ${ }^{27}$ The samples were stored in a dessicator to prevent oxidation of the surface prior to insertion in the ultrahigh vacuum system.

The samples were mounted on a tantalum-tungsten manipulator in an ultrahigh-vacuum chamber with a base pressure of $2 \times 10^{-10}$ Torr. A tungsten heating filament was placed behind the samples for radiative or electron beam heating and the sample temperature was monitored with a type- $K$ thermocouple. The samples were cleaned with Ar ion bombardment, and the surface composition was monitored with Auger electron spectroscopy (AES). The Auger electrons are created by an incident electron beam with an energy of $2.0 \mathrm{keV}$ and a current of $0.2 \mu \mathrm{A}$ at an angle of $60^{\circ}$ to the sample normal.

The Auger and photoemitted electrons are analyzed 
with a $90^{\circ}$ electrostatic sector with a mean radius of $117 \mathrm{~mm}$ manufactured by Surface/Interface Inc. The energy-analyzer front-end optics collects electrons emitted within a solid $\pm 20^{\circ}$ cone, collimates them with a Pierre electron lens system, and injects them into the energy analyzer. The analyzer is operated in the constant retardation-ratio mode, and the pass energy $E_{\text {pass }}$ of the electrons through the $90^{\circ}$ sector is one-fourth the initial kinetic energy. The $90^{\circ}$ sector is configured as half of a hemispherical analyzer and the electron-energy resolution $\Delta E$ is determined by the ratio of the radii of the exit aperture $r$ and the mean radius, $\Delta E / E_{\mathrm{pass}}=$ $r(\mathrm{~mm}) / 117$. For the AES data presented below, the exit aperture is $3.5 \mathrm{~mm}$, the spectra are accumulated in the pulse counting mode, the derivative is obtained numerically, and the energy resolution at $400 \mathrm{eV}$ initial kinetic energy (near the nitrogen Auger peak) is $1.28 \mathrm{eV}$. The photemission data are obtained with an exit aperture of $1.5 \mathrm{~mm}$, and the electron-energy resolution at $19 \mathrm{eV}$ initial kinetic energy is $0.06 \mathrm{eV}$.

\section{B. Nitrogen addition}

A unique method of nitriding was employed in order to maintain the base pressure in the vacuum system. The surface was initially cleaned with several cycles of argon sputtering and annealing to $350^{\circ} \mathrm{C}$, and Auger and photoemission spectra of the parent compound were acquired. Auger spectra obtained during the nitriding process are shown in Fig. 3. The nitrogen ions were im-

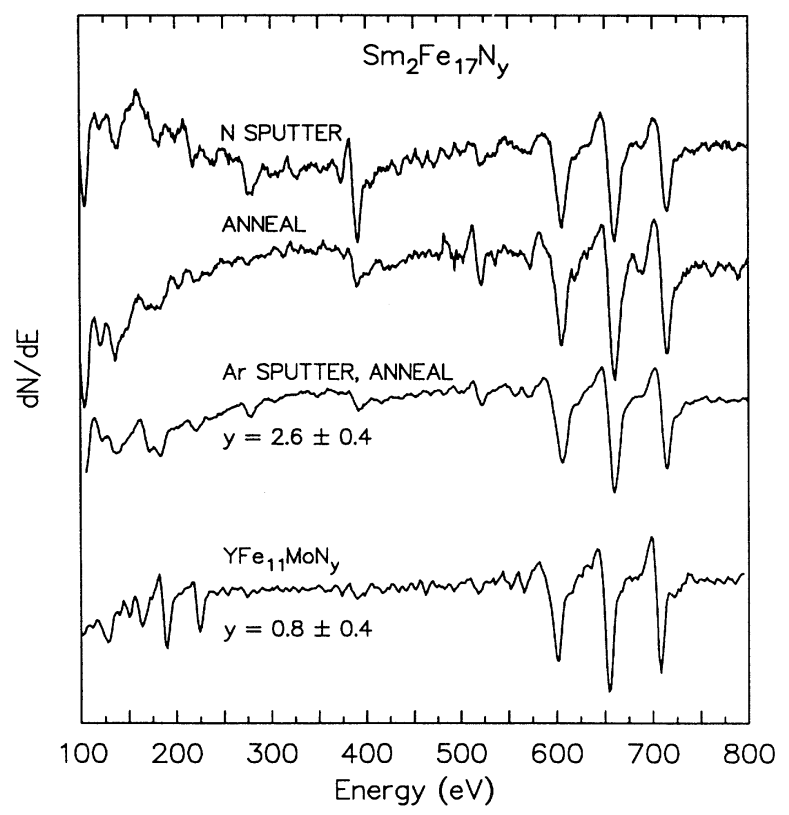

FIG. 3. Auger electron spectra for various steps in the nitriding process for $\mathrm{Sm}_{2} \mathrm{Fe}_{17}$. The nitrogen Auger energy is near $400 \mathrm{eV}$ and the $\mathrm{Fe}$ Auger energies are in the range of $600-700 \mathrm{eV}$. The nitrided $\mathrm{Sm}_{2} \mathrm{Fe}_{17}$ is compared to the nitrided $\mathrm{YFe}_{11} \mathrm{Mo}$. planted with a kinetic energy of $2 \mathrm{keV}$ and a current of $1 \times 10^{-6}$ amperes for $10 \mathrm{~min}$. The nitrogen ions were generated and accelerated with the same ion sputter gun used for argon sputter cleaning. Using the Auger sensitivity factors ${ }^{28}$ and normalizing the $\mathrm{Fe}$ to the formula value $\left(\mathrm{Sm}_{2} \mathrm{Fe}_{17} \mathrm{~N}_{x}\right)$, the $\mathrm{N}$ content is estimated to be $x=9.8 \pm 0.6$ after implantation. The nitrogen-rich surface region acts as a source of nitrogen for diffusion in subsequent anneals to $400^{\circ} \mathrm{C}$ for several minutes. The surface nitrogen concentration is reduced to $x=3.2 \pm 0.4$ after the sample was annealed in ultrahigh vacuum. The nitrogen-rich surface region is then removed with argon ion sputtering and the subsurface nitride layer was exposed. The nitrogen concentration $x$ was measured as a function of depth with argon ion sputtering and Auger spectroscopy. The subsurface nitrogen concentration was measured as $x=2.6 \pm 0.4$, and the nitride region was estimated to be $1.4 \mu \mathrm{m}$ thick. These results are similar to Kerr microscopy observations of the nitrogenation of grains by Mukai and Fujimoto. ${ }^{29}$ In their work, a surfacenitrided region approximately $1.5 \mu \mathrm{m}$ thick is present in grains of $\mathrm{Sm}_{2} \mathrm{Fe}_{17}$ after annealing at $500{ }^{\circ} \mathrm{C}$ in nitrogen for $1 \mathrm{~min}$.

The $\mathrm{NdFe}_{11} \mathrm{Ti}$ compound was nitrided using the same method, but unfortunately the Ti Auger line overlaps the $\mathrm{N}$ Auger line and an accurate measure of the $\mathrm{N}$ concentration in this compound was not obtained. The $\mathrm{YFe}_{12-x} \mathrm{Mo}_{x}$ compounds were also nitrided and the Auger spectrum from the nitrided compound is shown in Fig. 3. Normalizing the Fe content to the formula $\mathrm{YFe}_{11} \mathrm{MoN}_{y}$ a value of $y=0.8 \pm 0.4$ was obtained with AES. A similar value of reduced $\mathrm{N}$ concentration was observed in Co-based 1:12 compounds. ${ }^{30}$ The difference in nitrogen concentration for the two compounds supports the assertion that the bulk atomic structure is present up to the surface which is probed by AES and photoelectron spectroscopy (PES).

\section{Photoemission experiments}

The electronic structures of the samples were investigated with (PES) experiments carried out at the Synchrotron Radiation Center in Wisconsin. The energy distribution curves (EDCs) of the $\mathrm{Sm}_{2} \mathrm{Fe}_{17}$, $\mathrm{NdFe}_{11} \mathrm{Ti}$, and $\mathrm{YFe}_{12-x} \mathrm{Mo}_{x}$ compounds have been published previously, ${ }^{24,30,31}$ and the EDCs are included here with further data analysis on the DOS at $E_{F}$ as described below. The EDCs of Al-substituted $\mathrm{Sm}_{2} \mathrm{Fe}_{17-x} \mathrm{Al}_{x}$ compounds are included as a test of the spin-fluctuation theory. Spin-resolved EDCs from $\mathrm{Sm}_{2} \mathrm{Fe}_{17} \mathrm{~N}_{2.6}$ are compared to the calculated majority and minority DOS.

The photon-energy range used in the spin-integrated experiments was between 18 and $30 \mathrm{eV}$, and the spectra displayed here were obtained with $22.5 \mathrm{eV}$. In order to obtain a justifiable measure of the change in the DOS at $E_{F}, N_{\text {tot }}\left(E_{F}\right)$, the EDCs were obtained at several photon energies and the integrated area within several $\mathrm{eV}$ of the Fermi energy was normalized to the Fe concentration. Several integration energy ranges were considered, and the range from $-0.5 \mathrm{eV}$ to $2.0 \mathrm{eV}$ binding 
energy was taken to be dominated by the Fe $3 d$ electron emission. $N_{\text {tot }}\left(E_{F}\right)$ values were estimated by fitting the EDCs within $\pm 0.25 \mathrm{eV}$ of the Fermi energy with a roomtemperature Fermi function that was convolved with a Gaussian to represent the instrumental broadening. It is anticipated that the error bars for the data analysis will be substantial due to the difference in the structure
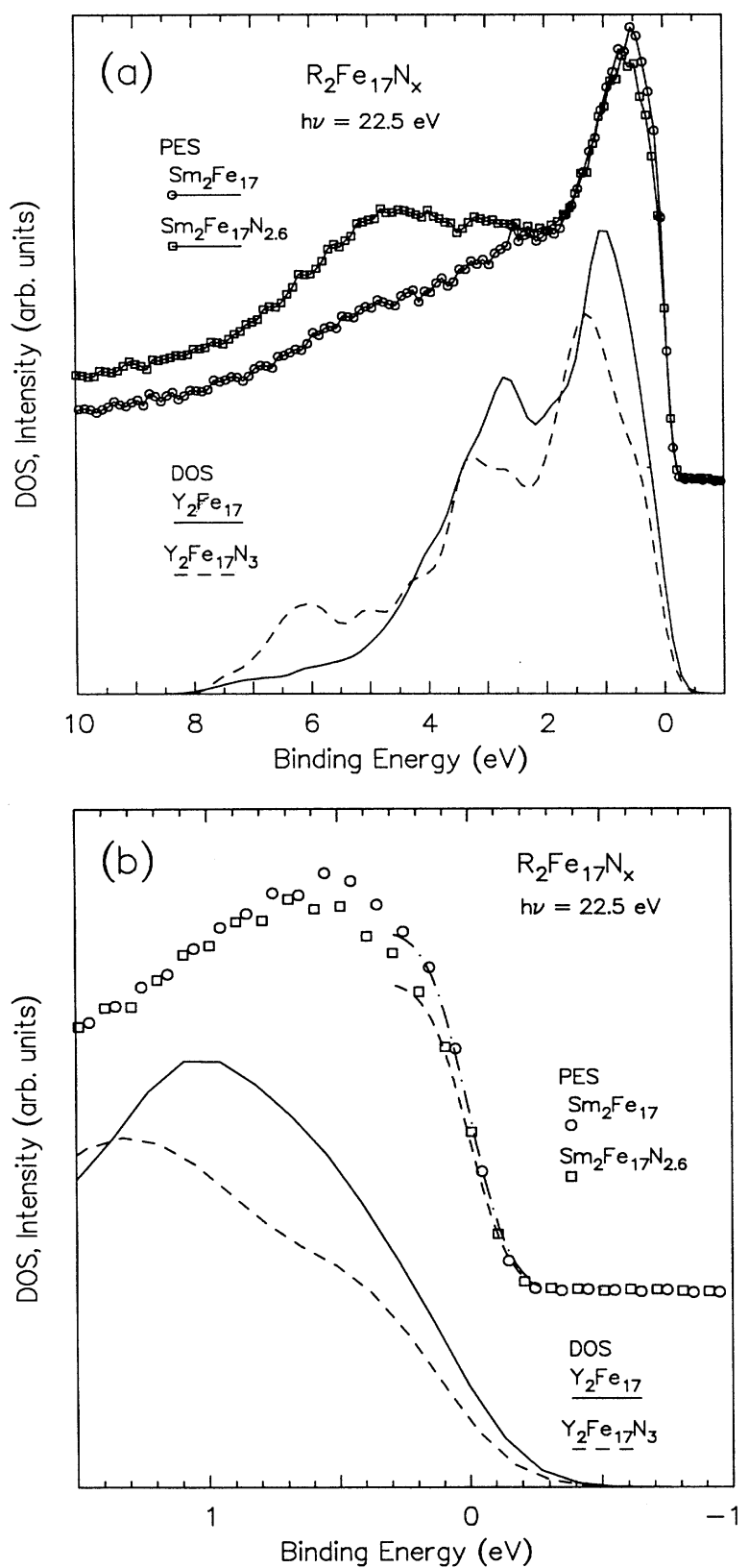

FIG. 4. (a) Upper curves: photoemission spectra for $\mathrm{Sm}_{2} \mathrm{Fe}_{17}$ and $\mathrm{Sm}_{2} \mathrm{Fe}_{17} \mathrm{~N}_{2.6}$ normalized as described in the text. Lower curves: calculated density of states for $\mathrm{Y}_{2} \mathrm{Fe}_{17}$ and $\mathrm{Y}_{2} \mathrm{Fe}_{17} \mathrm{~N}_{3}$. (b) Same data as in (a) with an expanded binding energy scale in the neighborhood of the Fermi energy. The dot-dashed and dashed lines on the experimental data are the fit of the convoluted Fermi function to the Fermi edge. causing a differing matrix element for photoemission and differing electron scattering.

The valence EDCs of the compounds are shown in Figs. 4-7 with calculated electronic structure where available. In all the spectra, the $\mathrm{Fe} 3 d$ band from 0 to $2 \mathrm{eV}$ is readily apparent and the second Fe feature near $2.5 \mathrm{eV}$ binding energy in the calculation is somewhat discernible
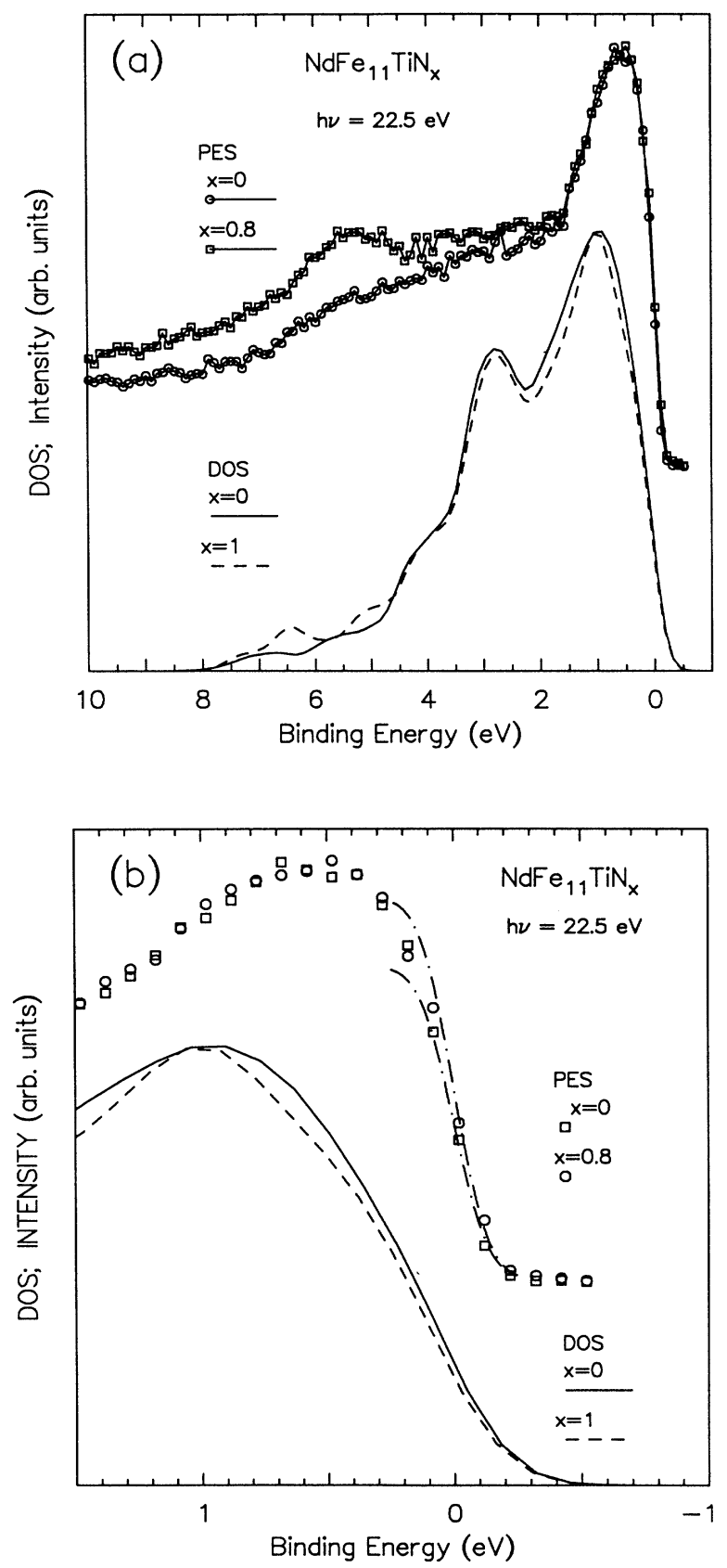

FIG. 5. (a) Upper curves: Photoemission spectra of $\mathrm{NdFe}_{11} \mathrm{Ti}$ and $\mathrm{NdFe}_{11} \mathrm{TiN}_{0.8}$. Lower curves: calculated density of states for the same compounds as noted. (b) Same data as in (a) with an expanded binding energy scale in the neighborhood of the Fermi energy. The dot-dashed lines are $\pm 10 \%$ of the best fit of the convoluted Fermi function. 
in the EDCs. The change in the $3 d$ bandwidth, an expected narrowing as the moment increases, cannot be discerned with the EDCs. The contribution due to nitrogen is observed in Figs. 4 and 5 at $\sim 5 \mathrm{eV}$, and the binding energy in the EDC is substantially lower than the calculated nitrogen binding energy. The most sub-
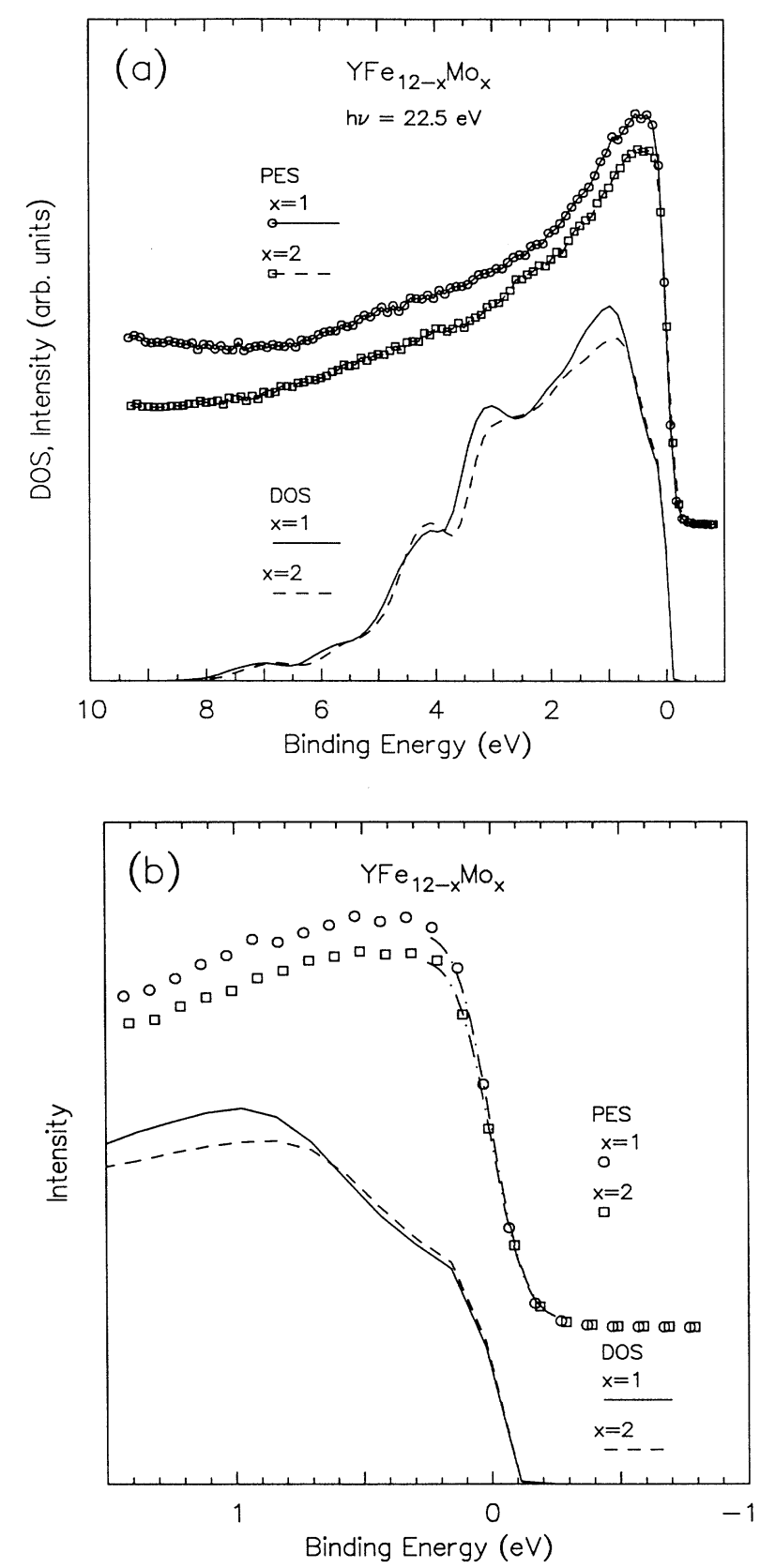

FIG. 6. (a) Upper curves: photoemission spectra for $\mathrm{YFe}_{11} \mathrm{Mo}$ and $\mathrm{YFe}_{10} \mathrm{Mo}_{2}$ normalized as described in the text. Lower curves: calculated density of states for $\mathrm{YFe}_{11} \mathrm{Mo}$ and $\mathrm{YFe}_{10} \mathrm{Mo}_{2}$. (b) Same data as in (a) with an expanded binding energy scale in the neighborhood of the Fermi energy. The dot-dashed lines on the experimental data are the fit of the convoluted Fermi function to the Fermi edge. stantial difference between the EDCs and the calculated DOS is that the EDC is a measure of a photoionized state which includes photoemission matrix elements, lifetime broadening, electron transport, and secondary electron phenomenon. The calculated DOS represents the ground state of the electronic structure and there is fair
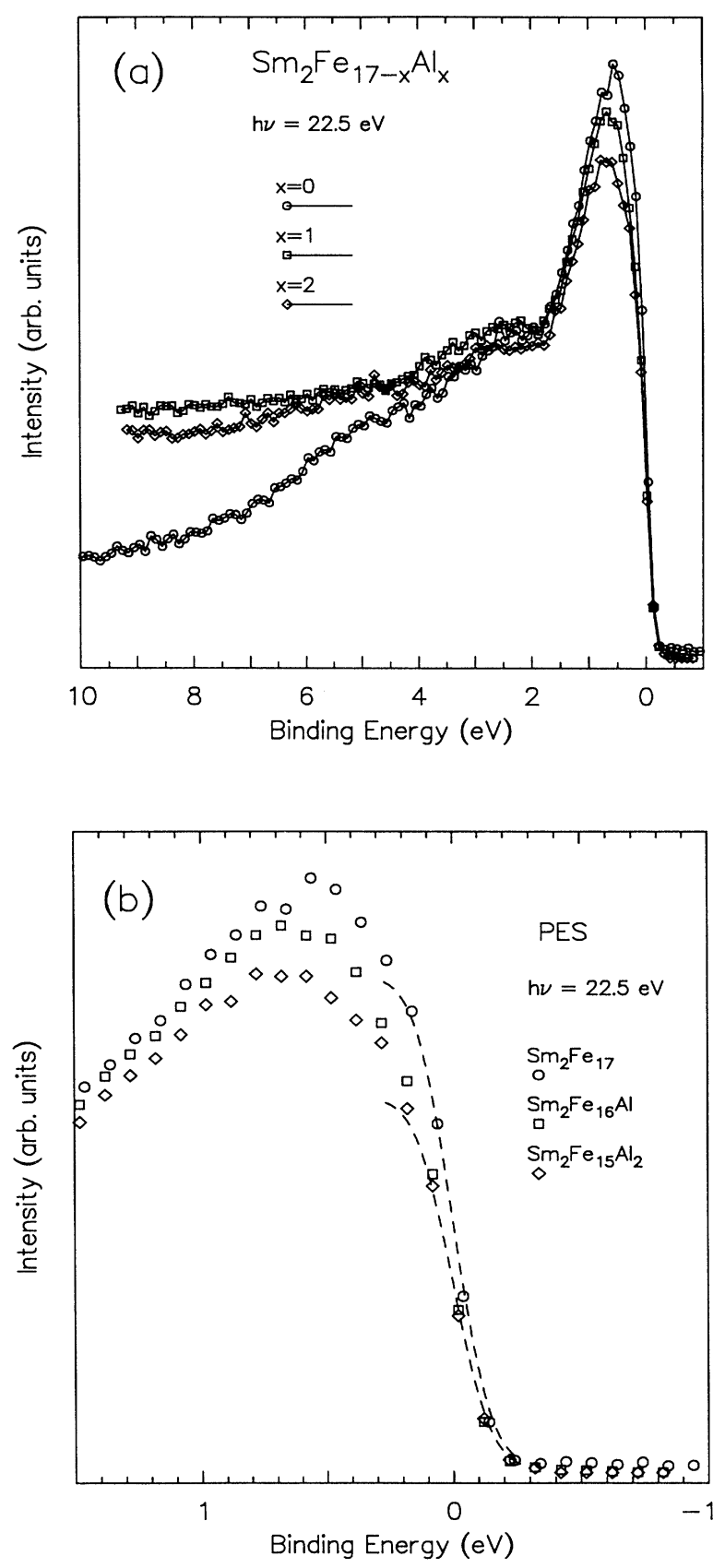

FIG. 7. (a) Photoemission spectra for $\mathrm{Sm}_{2} \mathrm{Fe}_{17-x} \mathrm{Al}_{x}$ with $x=0,1,2$. (b) Same data as in (a) with an expanded binding energy scale in the neighborhood of the Fermi energy. The dashed lines are the best fit of the convoluted Fermi function to the Fermi edge. 
agreement between the calculated DOS and the measured EDCs.

The lifetime broadening is due to the lifetime of the photocreated empty state; the larger the lifetime, the smaller the broadening. Models of the lifetime broadening generally assume that the lifetime is proportional to $\left(E_{B}-E_{F}\right)^{2}$, where $E_{B}$ is the binding energy. The lifetime is roughly determined by the number of states (with lower binding energy) available to fill the empty state, and the broadening at $E_{F}$ is generally assumed to be zero. The states at $E_{F}$ have a long lifetime, and the EDC at $E_{F}$ can be taken as a measure of the DOS at $E_{F}$. The EDCs and calculated DOS within $1.5 \mathrm{eV}$ of the Fermi energy are shown in Figs. 4(b)-7(b). In all the figures, the experimental Fermi edge is sharper than the calculated edge which has been broadened by a Gaussian to simulate instrumental response, and the peak position of the $3 d$ EDC bands occur at a substantially lower binding energy than the calculated $3 d$ DOS bands. Qualitatively the change in the EDCs in Figs. 4(b)-7(b) agree with the change in the calculated DOS. In Fig. 5(b), the upper and lower dot-dashed curves at $E_{F}$ are $\pm 10 \%$ error bars of the best fit to both sets of EDCs. The error for each individual EDC is estimated as $\pm 8 \%$ and the ratio of the EDCs at $E_{F}$ will include an error of $\pm 16 \%$. In all cases the EDCs and the DOS decrease as $T_{c}$ increases within experimental error. Quantitatively, the results of the fitting of the convoluted Fermi functions are given in Table I and compared to the calculated results. The relative change of the EDCs and the DOS for both nitrogen interstitial and substitutional modifications are in very good agreement, within the limit of the experimental error.

In Fig. 7(b) the modifications of the EDCs at $E_{F}$ are shown with the fitted convoluted Fermi function. The measured EDC at $E_{F}$ can be used with the enhanced susceptibility curve in Fig. 2 and the experimentally measured $M_{0}$ values to determine a ratio in $T_{c}$ for $\mathrm{Al}$ substitution which can be compared to experimentally determined $T_{c}$ ratio. The $T_{c}$ ratio determined by the spin-fluctuation model can be written

$$
\frac{T_{c}(x=2)}{T_{c}(x=0)}=\left(\frac{M_{0}(x=2)}{M_{0}(x=0)}\right)^{2} \frac{\chi_{0}(x=0)}{\chi_{0}(x=2)}
$$

TABLE I. Ratios of the calculated density of states at the Fermi energy and the fitted experimental energy distribution curve at the Fermi energy.

\begin{tabular}{ccc}
\hline \hline Compounds & Calculation & $\begin{array}{c}\text { Experiment } \\
\text { (error } \pm 0.16)\end{array}$ \\
\hline$\frac{R_{2} \mathrm{Fe}_{17} \mathrm{~N}_{3}}{R \mathrm{Fe}_{17}}$ & 0.75 & 0.85 \\
$\frac{\mathrm{NdFe}_{11} \mathrm{TiN}}{\mathrm{NdFe}}$ & $0.91 \mathrm{Ti}$ & 1.0 \\
$\frac{\mathrm{YFe}_{11} \mathrm{Mo}}{\mathrm{YFe}_{10} \mathrm{Mo}_{2}}$ & 0.96 & 1.07 \\
$\frac{\mathrm{Sm}_{2} \mathrm{Fe}_{15} \mathrm{Al}_{2}}{\mathrm{Sm}_{2} \mathrm{Fe}_{17}}$ & & 0.75 \\
\hline \hline
\end{tabular}
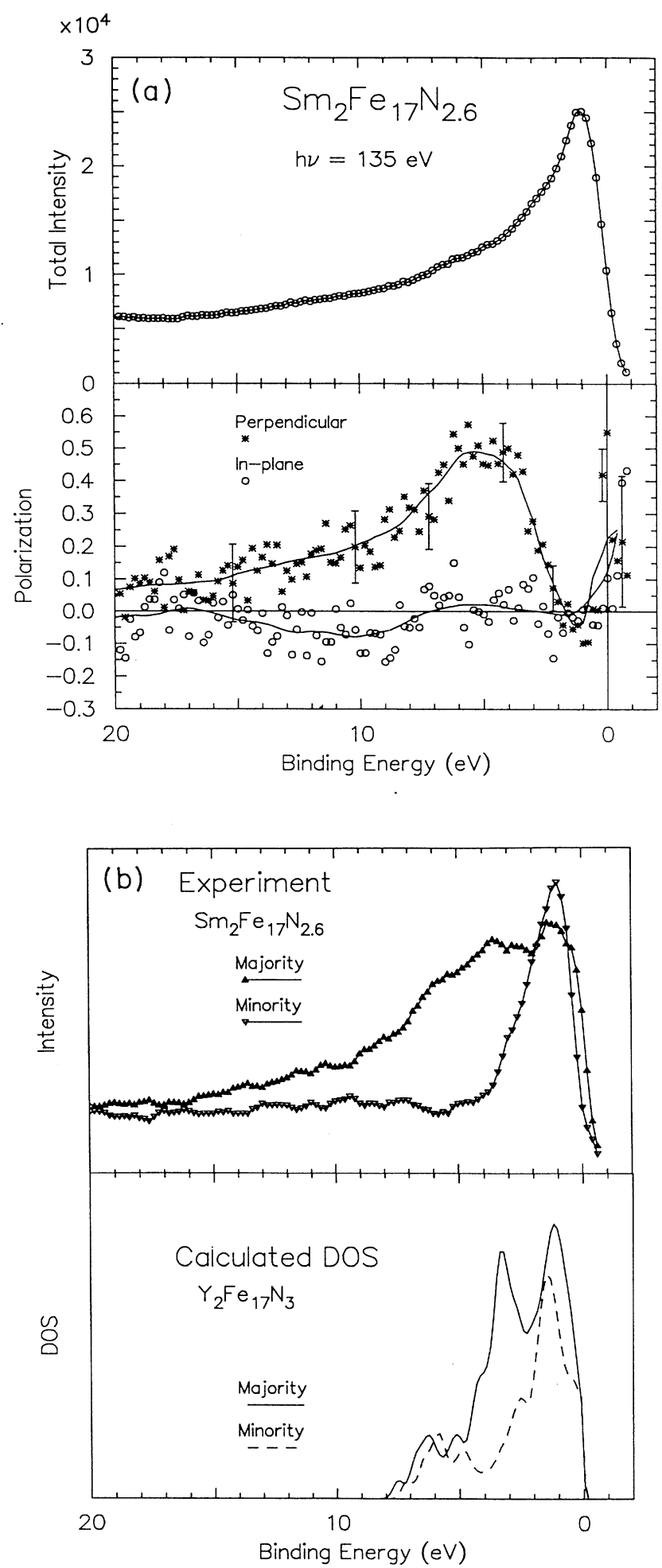

FIG. 8. (a) Spin-resolved photoemission of $\mathrm{Sm}_{2} \mathrm{Fe}_{17}$ obtained with a photon energy of $135 \mathrm{eV}$ showing the total intensity and the measured perpendicular and in-plane components of the polarization as a function of binding energy using a Sherman function of 0.05. (b) Comparison of the experimental and calculated majority- and minority-spin EDCs in the perpendicular orientation. 
where $x$ is the aluminum content, the zero-temperature moments are $M_{0}(0)=35.2 \mu_{B} /$ f.u., and $M_{0}(2)=$ $27.4 \mu_{B} /$ f.u. The enhanced susceptibility for the $\mathrm{Y}_{2} \mathrm{Fe}_{17}$ compound from Ref. 20 and plotted in Fig. 2 is $\chi_{0}(0)=$ $2.77 \mu_{B}^{2}$. Using this value with the change in EDCs at $E_{F}$ in Fig. $7(\mathrm{~b})$, the enhanced susceptibility for $x=2$ is $\chi_{0}(2)=1.55 \mu_{B}^{2}$. The experimentally determined ratio of the $T_{c}$ values is $1.16,{ }^{8}$ and using the above determined parameters the spin-fluctuation theory predicts a ratio of

$$
\frac{T_{c}(2)}{T_{c}(0)}=\left(\frac{27.4}{35.2}\right)^{2} \frac{2.77}{1.55}=(0.61)(1.79)=1.09
$$

which is within $6 \%$ of the experimentally determined $T_{c}$ ratio. Thus the spin-fluctuation model predicts the $T_{c}$ change of the substitutionally modified compound very well.

\section{Spin-resolved photoemission}

Spin-resolved photoemission of the $\mathrm{Sm}_{2} \mathrm{Fe}_{17} \mathrm{~N}_{2.6}$ valence band was obtained with a higher-intensity and higher-energy $(135 \mathrm{eV})$ photon beam on the Mark V monochromator at the synchrotron. The photon energy is below the $\mathrm{Sm} 4 d \rightarrow 4 f$ resonance, and the $\mathrm{EDC}$ is dominated by the $\mathrm{Fe} 3 d$ electrons. A diffuse-scattering spin analyzer with a Sherman function of 0.05 (Ref. 32) is used to measure two components of the photoelectron spin perpendicular and parallel to the surface. The $\mathrm{Sm}_{2} \mathrm{Fe}_{17}$ sample was mounted on a magnetic core and a magnetic field was applied to the sample. After nitriding the sample, the $c$ axis is the easy axis of magnetization and the polarizations perpendicular and parallel to the surface are presented in Fig. 8(a) with the total intensity. The statistical error is shown at several points on the polarization curves and the solid line is determined by a smoothing of the raw data and is intended to serve as a guide to the eye. The data are obtained from two EDCs from the four anodes in the spin analyzer and the instrumental asymmetry is removed using the data analysis described by Kessler. ${ }^{33}$ The in-plane component of polarization is zero within the statistical error, and the perpendicular polarization shows significant structure in the valence band. The polarization spectra show that the material is magnetized perpendicular to the surface which agrees with previous determination of the magnetic anisotropy of the nitrided $\mathrm{Sm}_{2} \mathrm{Fe}_{17}$ compounds.

The perpendicular polarized majority- and minorityspin components of the spectra obtained from the polarization and intensity data are shown in Fig. 8(b) with the spin-resolved DOS calculation. ${ }^{20}$ The minority-spin experimental data show a good agreement with the calculation and the majority-spin data exhibit a broad feature, and the net polarization is positive for binding energies up to $18 \mathrm{eV}$. The spin-resolved spectra in Fig. 8 near the Fermi energy show that the polarization is positive at $E_{F}$ in excellent agreement with the spin-resolved calculated DOS shown in the figure. In previous spin-resolved PES spectra from pure iron samples, ${ }^{34}$ the polarization is dominated by minority-spin electrons within $1 \mathrm{eV}$ of the Fermi energy.

\section{CONCLUSIONS}

The spin-fluctuation $T_{c}$ theory of Mohn and Wohlfarth has been applied to both interstitial and substitutional modifications of the $2: 17$ and 1:12 R-T compounds. The theory and experimental data support the spin-fluctuation model of $T_{c}$ as the model correctly predicts the change in $T_{c}$ as the compound is modified. From the calculated electronic structures, the enhanced susceptibility $\chi_{0}$ is linearly related to the total (spin-summed) DOS at $E_{F}$. This result has been applied to determine the change in $T_{c}$ of the Al-substituted $\mathrm{Sm}_{2} \mathrm{Fe}_{17-x} \mathrm{Al}_{x}$ compound with very good agreement between the model and the measured $T_{c}$ change. Spinresolved photoemission spectra from $\mathrm{Sm}_{2} \mathrm{Fe}_{17}$ have shown that the magnetization is perpendicular to the surface and the spin-resolved electronic structure agrees with the calculated DOS. The $T_{c}$ modification of the Fe-based $R$ Fe compounds have been well modeled with the spinfluctuation model. Substitutional alloying or interstitial nitriding or carbiding of the parent binary or ternary compounds has important practical implications in certain cases for permanent-magnet materials. The understanding achieved in the present series of measurements and calculations is a first step in developing a more detailed understanding based on local-environment effects, moment instabilities, and spin-polarized disordered alloying effects. These considerations will be important for future studies of these and related materials.

\section{ACKNOWLEDGMENTS}

This work was supported by the U.S. DOE under Grant No. DE-FG02-86ER45262, the National Science Foundation under Grant No. OSR-9255225, Nebraska Energy Office, and Cornell Supercomputing Facility. The authors thank the staff of the Synchrotron Radiation Center in Wisconsin which is supported by the National Science Foundation.
${ }^{1}$ J. F. Herbst, Rev. Mod. Phys. 63, 819 (1991), and references therein.

${ }^{2}$ K. J. Strnat, in Ferromagnetic Materials, edited by E. P. Wohlfarth and K. H. J. Buschow (North-Holland, Amsterdam, 1989), Vol. 4, p. 131.

${ }^{3}$ K. H. J. Buschow, in Ferromagnetic Materials, edited by E. P. Wohlfarth and K. H. J. Buschow (North-Holland, Ams- terdam, 1986), Vol. 1, p. 297.

${ }^{4}$ J. M. D. Coey and H. Sun, J. Magn. Magn. Mater. 87, L251 (1990); H. Sun, J. M. D. Coey, Y. Otani, and D. P. R. Hurley, J. Phys. Condens. Matter 2, 6465 (1990).

${ }^{5} \mathrm{D}$. McNeely and H. Oesterreicher, J. Less-Common Met. 44, 183 (1976).

${ }^{6}$ T. H. Jacobs, K. H. J. Buschow, G. F. Zhou, X. Li, and F. 
R. de Boer, J. Magn. Magn Mater. 116, 220 (1992).

${ }^{7}$ Bo-Ping Hu, Xiao-Lei Rao, Jian-Min Xu, Gui-Chuan Liu, Fei Cao, Xiao-Lin Dong, Hua Li, Lin Yin, and Zhong-Ren Zhao, J. Magn. Magn. Mater. 114, 138 (1992).

${ }^{8} \mathrm{Z}$. Wang and R. A. Dunlap, J. Phys. Condens. Matter 5, 2407 (1993).

${ }^{9}$ J. M. D. Coey, in Ferromagnetic Materials, edited by E. P. Wohlfarth and K. H. J. Buschow (North-Holland, Amsterdam, 1991), Vol. 6, p. 1.

${ }^{10}$ Hong Sun, M. Akayama, K. Tatami, and H. Fujii, Physica B 183, 33 (1993).

${ }^{11}$ D. B. de Mooij and K. H. J. Buschow, J. Less-Common. Met. 136, 207 (1988).

${ }^{12}$ Hong Sun, J. M. D. Coey, Y. Otani, and D. P. F. Hurley, J. Phys. Condens. Matter 2, 6465 (1990).

${ }^{13}$ Qi-nuan Qi, Hong Sun, R. Skomski, and J. M. D. Coey, Phys. Rev. B 45, 12278 (1992).

${ }^{14}$ M. Katter, J. Wecker, L. Schultz, and R. Grossinger, J. Magn. Magn. Mater. 92, L14 (1990).

${ }^{15}$ P. Mohn and E. P. Wohlfarth, J. Phys. F 17, 2421 (1987).

${ }^{16} \mathrm{John}$ B. Goodenough, Magnetism and the Chemical Bond (Interscience, New York, 1963), pp. 78-80.

${ }^{17}$ Chih-Wen Chen, Magnetism and Metallurgy of Soft Magnetic Materials (Dover, New York, 1986).

${ }^{18}$ D. P. Middleton and K. H. J. Buschow, J. Alloys Compounds 206, L1 (1994).

${ }^{19}$ E. P. Wohlfarth, Phys. Lett. 3, 17 (1962).

${ }^{20}$ S. S. Jaswal, W. B. Yelon, G. C. Hadjipanayis, Y. Z. Wang, and D. J. Sellmyer, Phys. Rev. Lett. 67, 644 (1991).

${ }^{21}$ S. S. Jaswal, Phys. Rev. B 48, 6156 (1993).

${ }^{22}$ H. K. Skriver, in The LMTO Method, edited by M. Cardona,
P. Fulde, and H.-J. Queisser, Springer Series in Solid State Sciences Vol. 41 (Springer-Verlag, New York, 1984).

${ }^{23}$ M. Katter, J. Wecker, C. Kuhurt, L. Schultz, and R. Grossinger, J. Magn. Magn. Mater. 114, 35 (1992).

${ }^{24}$ A. S. Fernando, J. P. Woods, S. S. Jaswal, B. M. Patterson, D. Welipitiya, and D. J. Sellmyer, J. Appl. Phys. 75, 6303 (1994).

${ }^{25}$ B.-P. Hu, H.-S. Li, J. P. Gavigan, and J. M. D. Coey, J. Phys. Condens. Matter 1, 755 (1989).

${ }^{26}$ A. E. Platts, I. R. Harris, and J. M. D. Coey, J. Alloys Compounds 185, 251 (1992).

${ }^{27}$ J. M. D. Coey (private communication).

${ }^{28}$ Lawerence E. Davis, Noel C. MacDonald, Paul W. Palmberg, Gerald E. Riach, and Roland E. Weber, Handbook of Auger Electron Spectroscopy (Perkin Elmer, Eden Prairie, MN, 1978).

${ }^{29}$ Toshio Mukai and Tatsuo Fujimoto, J. Magn. Magn. Mater. 103, 165 (1992).

${ }^{30}$ A. S. Fernando, J. P. Woods, S. S. Jaswal, B. M. Patterson, D. Welipitiya, A. S. Nazareth, and D. J. Sellmyer, J. Appl. Phys. 73, 6919 (1993).

${ }^{31}$ J. P. Woods, A. S. Fernando, S. S. Jaswal, B. M. Patterson, D. Welipitiya, and D. J. Sellmyer, J. Appl. Phys. 73, 6913 (1993).

${ }^{32}$ D. G. Van Campen, R. J. Pouliot, and L. E. Klebanoff, Phys. Rev. B 48, 17533 (1993).

${ }^{33}$ Joachim Kessler, Polarized Electrons (Springer-Verlag, New York, 1985), p. 234.

${ }^{34}$ E. Kisker, in Spin- and Angle-Resolved Photoemission from Ferromagnets, edited by R. Feder (World Scientific, Singapore, 1985). 